\title{
Embolization of Pancreaticoduodenal Artery Pseudoaneurysm Using Glubran in a Patient with SARS CoV-2
}

\author{
Matteo Renzulli MD ${ }^{1,2}$, Daniele Spinelli MD ${ }^{1,2}$, Nicolò Brandi MD ${ }^{1,2}$ \\ and Rita Golfieri MD ${ }^{1,2}$ \\ 'Department of Radiology, IRCCS Azienda Ospedaliero-Universitaria di Bologna, Via Albertoni 15, Bologna, Italy \\ ${ }^{2}$ Radiology Unit, Department of Experimental, Diagnostic and Specialized Medicine, Sant'Orsola Hospital, \\ University of Bologna, Bologna, Italy
}

Keywords: Embolization; Pseudoaneurysm; Endovascular Procedure; Bleeding

Gastroduodenal arterial pseudoaneurysms could potentially evolve into life-threatening vascular complications. Urgent treatment is mandatory in symptomatic patients but might also be indicated even in those with asymptomatic pseudoaneurysms detected incidentally on imaging because of the high risk of rupture [1-3]. During recent years, endovascular methods for treatment of these pathologies has been preferred because of their minimal invasiveness, great efficacy, and larger applicability in patients unfit for surgical procedures [1-4].

On $10^{\text {th }}$ of January 2021, a 72-year-old woman presented at our hospital's emergency department with mild respiratory symptoms characterized by asthenia, mild fever associated with pallor, and generalized pain in the upper abdomen. The blood count performed at that time indicated severe anemia with $7.7 \mathrm{~g} / \mathrm{l}$ of hemoglobin and $12 \times 10^{3}$ leukocytosis; in addition, the patient tested positive for SARS CoV-2. A computed tomography was performed, which showed a $13-\mathrm{mm}$ pseudoaneurysm of the pancreaticoduodenal artery

\section{Corresponding author:}

Matteo Renzulli MD, Department of Radiology, IRCCS Azienda Ospedaliero-Universitaria di Bologna, Via Albertoni 15, Bologna, Italy.

Email: matteo.renzulli@aosp.bo.it

(C) 2021 CC BY 4.0 - in cooperation with Depts. of Cardiothoracic/ Vascular Surgery, General Surgery and Anesthesia, Örebro University Hospital and Örebro University, Sweden with a sign of rupture (voluminous blood peritoneal collection) (Figure 1). Owing to the poor clinical condition, it was decided to proceed immediately with endovascular treatment. The pseudoaneurysm was typically alimented by vessels deriving from the gastroduodenal and inferior pancreatoduodenal arteries. Distally, these arteries were tiny and, therefore, it was decided to embolize by injecting Glubran (Figure 2); this embolizing agent corresponds chemically to N-butyl-2-cyanoacrylate and is a permanent liquid embolic material that shows several advantages when compared with other embolic agents in gastrointestinal hemorrhage. It has low viscosity and polymerizes at a low temperature, thus allowing rapid and permanent embolization. Moreover, it can be injected through a microcatheter into small arteries, which is often too difficult to do. N-butyl-2-cyanoacrylate can also allow simultaneous embolization of collateral vessels connected to the hemorrhagic focus, which can prevent recurrent bleeding from retrograde collateral flow; moreover, it acts independently from the coagulation cascade and, therefore, this material is successfully employed even in patients suffering from severe coagulation issues [4]. A CT performed at $36 \mathrm{~h}$ after the procedure confirmed the treatment's efficacy, with no signs of intestinal/pancreatic ischemia and with hematoma reduction (Figure 3). The origin of this pseudoaneurysm was not related to the presence of acute pancreatitis (from clinical, radiological and laboratory points of view), and we were aware of no previous endovascular treatments. Moreover, several vascular anomalies were noted during the procedure that could be 

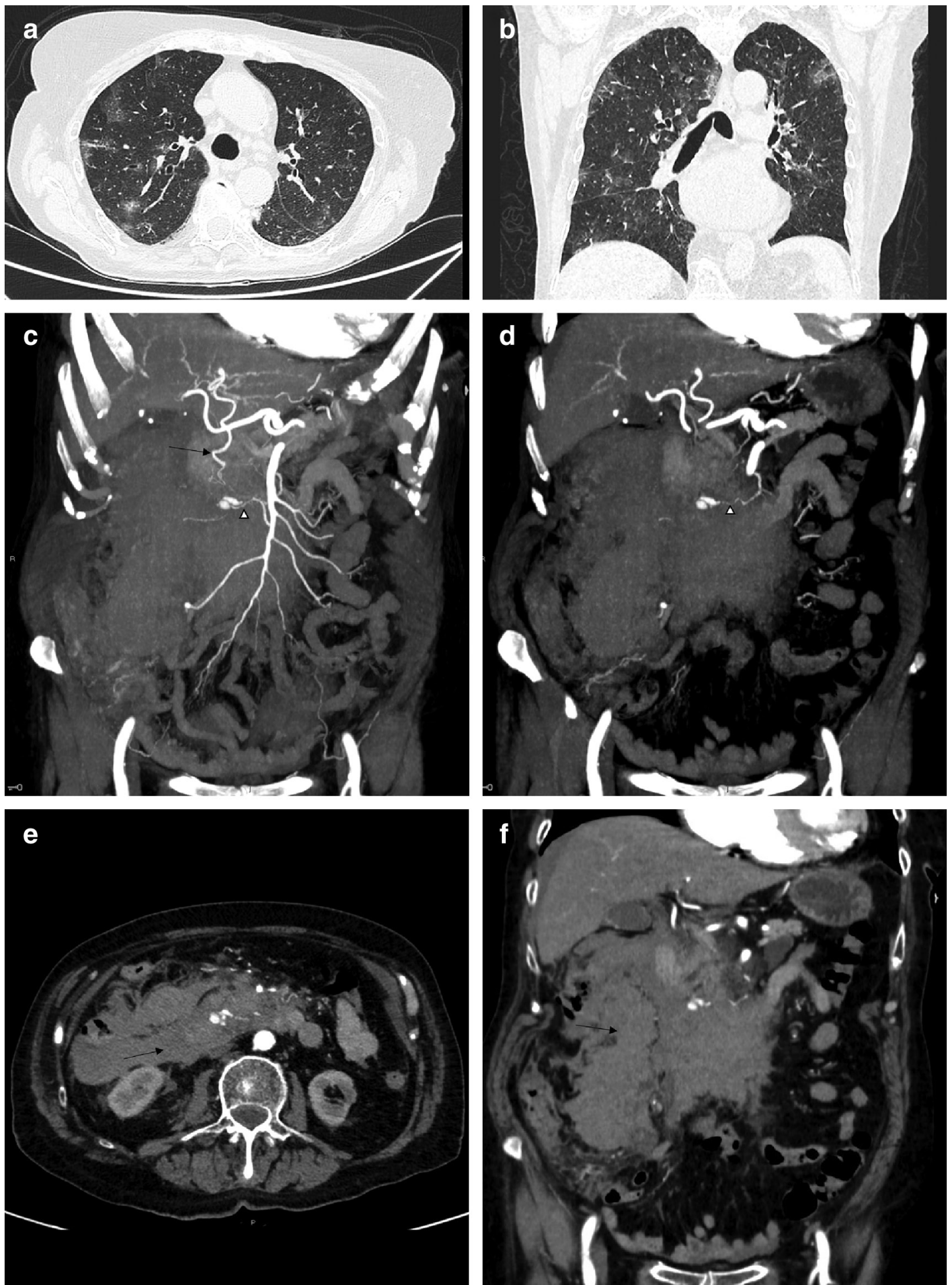

Figure 1 Chest computed tomography (CT) in axial (a) and coronal (b) planes showing bilateral, multifocal rounded subpleural "ground-glass" opacities compatible with SARS-CoV-2 infection. CT of the abdomen in coronal plane demonstrating the ruptured pseudoaneurysm of the pancreatoduodenal artery, fed by the gastroduodenal artery (black arrow; c) and the lower branch of pancreatoduodenal artery (arrowheads; c, d). CT of the abdomen in axial (e) and coronal (f) planes highlighting a voluminous intra-abdominal hematoma (black arrows) due to the pseudoaneurysm rupture. 


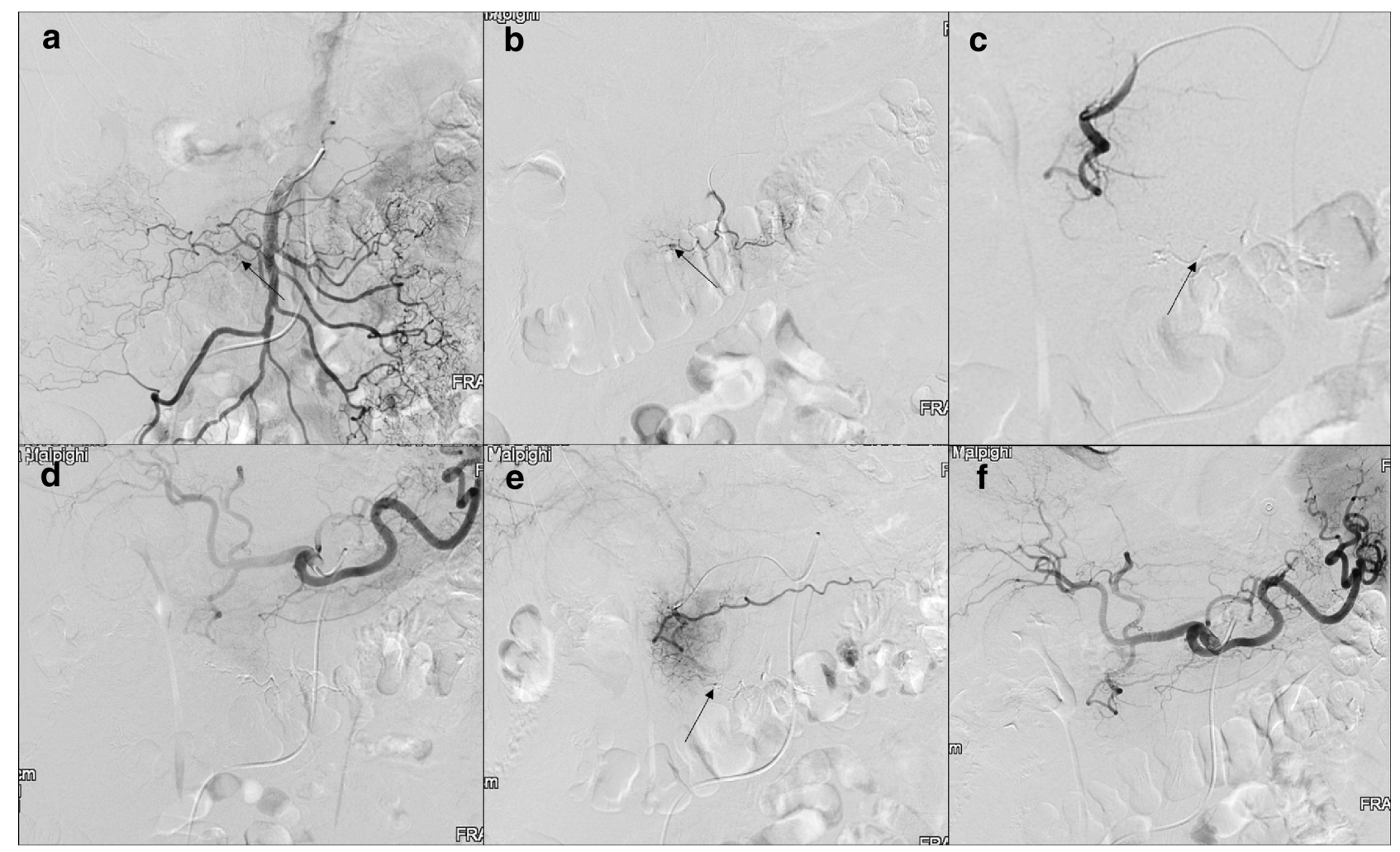

Figure 2 Angiographic study of the superior mesenteric artery (a) revealing the pseudoaneurysm of the pancreaticoduodenal artery (black arrow), more evident after selective catheterization of the inferior pancreatoduodenal artery (black arrow; b). The Glubran utilized to embolize the pseudoaneurysm is well detectable (black arrow). The proximal angiographic study of the celiac trunk does not show a feeding vessel of the pseudoaneurysm (d), well detectable after distal catheterization of the gastroduodenal artery (black arrow; e). This small vessel was also embolized finally excluding all pseudoaneurysm feedings (f).

attributable to a vasculitis related to SARS CoV-2 infection. The hospitalization was prolonged due to viral infection, with no signs of anemia.

\section{Ethics Statement}

(1) All the authors mentioned in the manuscript have agreed to authorship, read and approved the manuscript, and given consent for submission and subsequent publication of the manuscript.

(2) The authors declare that they have read and abided by the JEVTM statement of ethical standards including rules of informed consent and ethical committee approval as stated in the article.

\section{Conflicts of Interest}

The authors declare that they have no conflicts of interest.

\section{Funding}

The authors received no financial support for the research, authorship, and/or publication of this article.

\section{Author Contributions}

Idea conception: MR, DS. Data acquisition: MR, DS, NB. Manuscript preparation: MR, DS. Manuscript editing and reviewing: all authors. Quality control of 

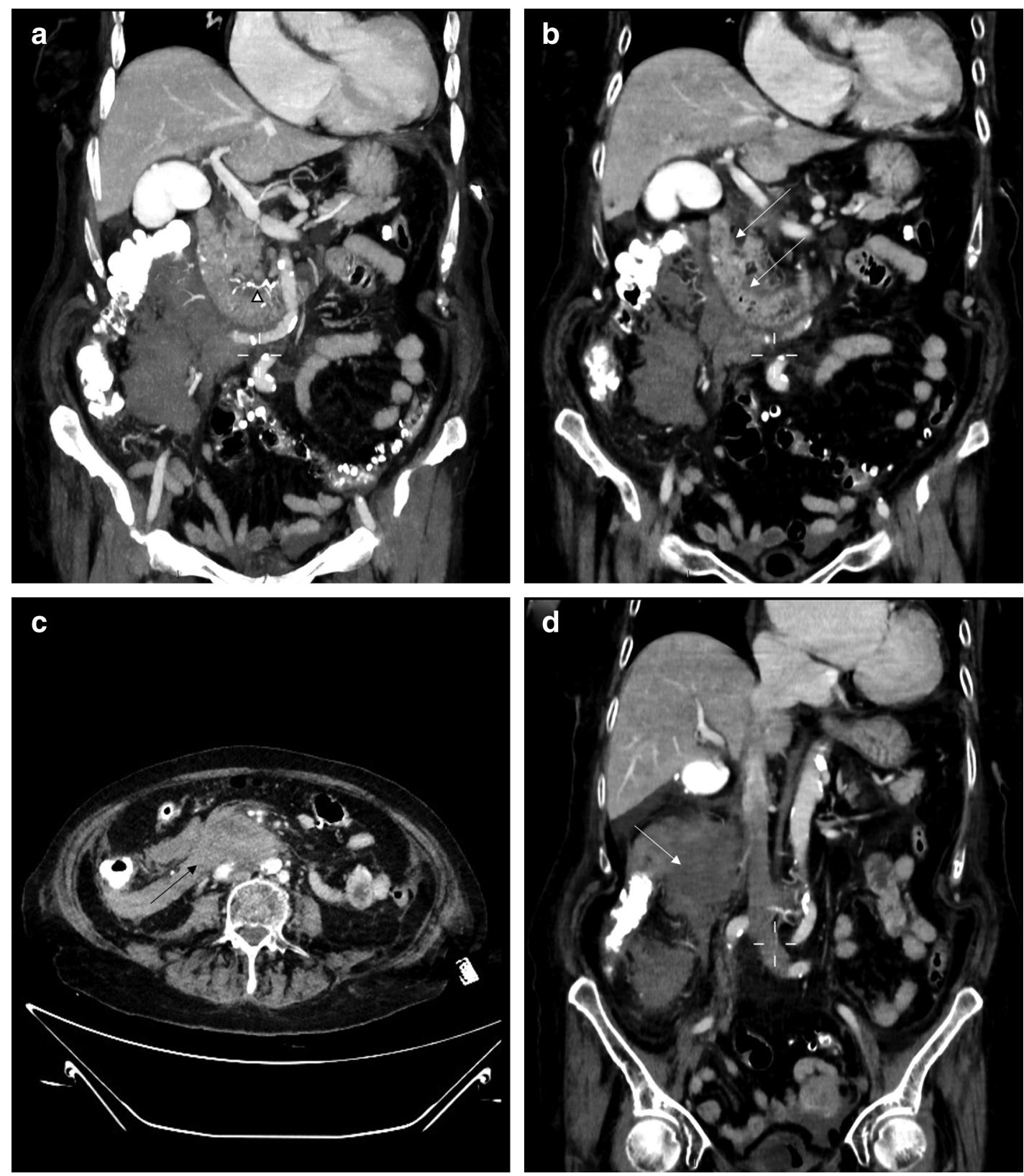

Figure 3 Coronal computed tomography (CT) of the abdomen showing the Glubran used for the pseudoaneurysm embolization (arrowhead; a), with no signs of ischemia of the duodenal walls (arrows; b). CT of the abdomen showing a huge reduction of the peritoneal hematoma in axial (arrow in c) and coronal (arrow in d) planes when compared with those in images in Figure $1 e, f$.

data: all authors. All authors read and approved the final manuscript.

\section{REFERENCES}

[1] Gorsi U, Agarwal V, Nair V, et al. Endovascular and percutaneous transabdominal embolisation of pseudoaneurysms in pancreatitis: an experience from a tertiary-care referral centre. Clin Radiol. 2021; In Press. doi: 10.1016/j.crad.2020.12.016.
[2] Barrionuevo P, Malas MB, Nejim B, et al. A systematic review and meta-analysis of the management of visceral artery aneurysms. J Vasc Surg. 2020;72(1S):40S-45S.

[3] Binetti M, Lauro A, Golfieri R, et al. False in name only-gastroduodenal artery pseudoaneurysm in a recurrently bleeding patient: case report and literature review. Dig Dis Sci. 2019;64(11):3086-91.

[4] Renzulli M, Mosconi C, Ascanio S, et al. Young women with acute lower gastrointestinal bleeding. Ann Emerg Med. 2016;68(2):e55-6. 